\title{
The Genius of Alan Turing: The Computing Classical Model
}

\author{
Luís Homem
}

\begin{abstract}
This paper aims to examine the basis of Calculus and computus from first philosophical principles, having a focus on the internal representations and acts of spontaneity, proper of genius that the concept of creativity is affiliate with. Our guiding author is Alan Turing and we will enquire closely the computing classical model. The paper explores the traditions of computing and philosophy, theorizing about the question of bio-machine hybrids in relation with imagination, the form of representation most free from nature. The first section is called calculus et computus. It examines the developments associated with the notions of algorithm, function and rule. In the second section the faculty of imagining is addressed through the abbreviated table, hoping to identify the boundaries both theoretical and practical of the computing classical model, following the seminal paper on computable numbers with Application to the Entscheidungs Problem (1936). We show how much hybridization of ideas fostered by both traditions was to find a place in the imaginary of artificial intelligence. Flanked by intuitions and concepts, imagination, the synthesis of reproduction, is capable of discerning about cosmos through bios and computus, so powerfully as if it sketched ideas in images, as the Turing machine clearly exemplifies.
\end{abstract}

Index Terms-Artificial intelligence, bio-machine hybrids, calculus et computus, computing classical model, creativity.

\section{INTRODUCTION}

The computing classical model has sprung universally and intrinsically from the Turing machine and the universal Turing machine. We aim to trace back the constituent phases of this convolution, by presenting at last a panoptical and functional plane of the computus realization, which has, by the passage from incompleteness to effective calculability, endeavored the faculty of imagination and genius towards the critical limits of cosmogenesis.

\section{Calculus et Computus}

\section{A. From Anthropocentric Humanism to "M-Configurations"}

Muhammad Ibn Musa Al-Khwarizmi (850 AD to 780 AD), the Muslim mathematician who first wrote about the system of hindu-arabic numerals and from whose book Kitab al-Jabr wa al-muqabalah comes the term "algebra", was also the source of the term "algorithm". The term "function" is a key definition as well.

Manuscript received September 2, 2013; revsied November 4, 2013.

Homem, Luís is with the Centre for Philosophy of Science of the University of Lisbon, Faculdade de Ciências da Universidade de Lisboa, Campo Grande, Edifício C4, $3^{\circ}$ piso, Sala 4.3.24 1749-016 Lisboa, Portugal (e-mail:1mhomem@yahoo.com).
Kant outlined in a wholly rationalist and empiricist way the importance of the rule in the sphere of cognition of human reason, as it metaphorically swerves, like a curve, in a very physicist metaphor, between questions called upon to consider due to its nature, but which cannot be answered. This curve often drags one specifically dynamics metaphor, as this curve is, of course, of a special tension.

One such example is to be found in the Critique of Pure Reason when the author from Königsberg analyses the proposition "all objects are beside each other in space", proceeding to the following:

"'All objects are beside each other in space', is valid only under the limitation that these things are taken as objects of our sensuous intuition. But if I join the condition to the conception and say, 'all things, as external phenomena, are beside each other in space', then the rule is valid universally, and without any limitation [1]."

Kant distinguishes on the one hand, validity under the limitation of objects as part of our sensuous intuition, and on the other hand, universal validity. We shall, henceforth, retain the idea of a curve that slopes, and that it's peculiar tension aggregates on the base a universal validity, and validity alone on the top, as it were in another section of the spectrum. In this context the line of tension is human reasoning itself. Unlike our daily experience, in this case universality belongs to the base and validity only to the top section of the spectrum. That is why it is associated with criticism.

This natural antinomy is not what we consider a rule, though. A rule is, more justifiably, a sort of natural critical admonition of human reasoning, instructing to experience. In the Cambridge Companion to Kant and Modern Philosophy we are alerted to the fact that Kant in the heart of the transcendental deduction (the Critique of Pure Reason, Second Edition) considered that even the pure concepts of understanding, such as of mathematics, applied directly to intuition and if one introduces the concept of quantity, it will provide cognition only insofar as there is experience, i.e., empirical intuitions:

"(...) he reminds us of his central theme about empirical knowledge, that the understanding must be "the source of the principles in accordance with which everything (that can even come before us as an object) necessarily stands under rules" [2].

Kant's Intuitionism was thus Empiricist.

Gödel's Intuitionism, on the contrary, was maybe only empiricist to the extent of being realistic in another way.

However, what is under the spotlight is the rule and there is a common denominator in both conceptions, namely what is axiomatically in accordance with the source of principles that 
constitutes understanding. A rule is axiomatically akin to principles and by definition it is of virtually infinite derivability, if proof is not possible.

Remembering that all intuitions are of extensive magnitudes, Kant, who hold a functionalist view of the Mind, nevertheless opposed axioms of intuition (Kant, I., Critique of Pure Reason [A162/B202]) (connected with the categories of Unity, Plurality and Totality) to axioms of mathematics, as these are by definition synthetic a priori and valid according to pure concepts. We could say that accordingly to Kant axioms of mathematics were necessarily related to knowledge (not to experience but intuition only) and axioms of intuition necessarily related to experience (and not necessarily to knowledge).

What Kant acknowledged as axioms of philosophy (Kant, I., Critique of Pure Reason, [A733/B761]) was essentially a mechanism of proof, a universal deduction of enlightenment, limited to Criticism, and exceptionally exact about the referred curve and announcement of antinomies.

The Aufklärung period heralded Industrialization and Industrialization did so with computation. Yet, in terms of anticipating the established model of computation through Alan Turing's paper On computable numbers with an Application to the Entscheidungs Problem (1936) the idea of calculable by finite means unfolded a dramatic transformation, profound enough to be called of new aesthetic perception, beyond any historical cultural views. Kant's idea on future metaphysics was still captive of the medieval conceptual-frame that admitted figures like angels.

Moreover, it was strongly hierarchical and, referring to one of the axioms of intuition, totally humanist.

That is to say, if we were to imagine humanity as a domain, according to Kant, there had to be an irrevocable ascent, with knowledge developing as a growing function in that domain, with the sum of those arbitrary units of knowledge being the ideal of mankind. This was recognized in different fashion in the contemporaneous era of computation by some philosophers, but in the totality of the concept could not be appreciated fully in Kant's time abridging the auxiliary Motus of machinery, little less with an outer-empowerment of man from alien, unperceptive, automated means. The extension of man by the computing power of modern and forthcoming events was hardly foreseeable even by a genius as the philosopher from Königsberg was.

It's ironic too that such an outstanding Platonist and classical ancient world representative as Gödel was, as a mathematician and a logician, to make a major contribution to computation and programming languages, generally by the apagogic method and rebound effect of proof. But there is inevitably a learning process at the birth of every new philosophy and artificial intelligence (Ai) did suffer some setbacks, for example the shift from strong Ai to weak Ai.

This factor, as well as artificial intelligence being considered historically a branch of computer science and not the reverse, serves to underline the huge influence of Platonism in western philosophy, which for example forced whitehead to consider European philosophy a footnote of Plato. Prior to Kant's death (1804) in the dawn of XIX ${ }^{\text {th }}$ century, we can look back to computata and automaton history, from Antikythera to Frederick ii (the great) of Prussia, whose patronage of arts, science and religious tolerance included the establishment of the Prussian (Berlin) academy of sciences. This supported such proponents of knowledge as Kant himself, but more importantly perhaps french philosophers (French was academy's official language), such as Encyclopedian D'Alembert, Condillac, Maupertuis and the autor of L'Homme Machine la Mettrie. Kant's pre-Industrialism Weltanschauung was, nevertheless, extremely important epistemologically in respect of the curve where mechanism joined machinery.

Innovative machinery such as the spinning jenny in the wool industry, the cotton gin and jacquard's loom, the water frame and moving factory cogs, the steam engine, and even the discovery of electricity by Volta, were all contemporary to Kant. In respect to Kant, our attention should lie more on the textile machinery than electricity. Kant knew and foresaw the practical implications of the textile machinery engineering power but not quite of electricity, even though Kant was miles away from England, the arena not so much of metaphysical debate, but of machinery debut.

It is the advent of textile machinery and electricity that together provide a first glance of the genesis of computation, along the future lines of a Turing machine.

It should not be forgotten that jacquard's loom machinery used punched cards, just the same as early digital $\mathrm{XX}^{\text {th }}$ century computers.

Babbage and Lovelace together can be considered as the first in persona modus of the conceptual pair of hardware and software. This was sort of the empirical postulate that history found to give proper rise to computation akin to the future ideal Turing machine. Of course a generalized theoretical understanding of magnetism and electricity had yet to be developed. Overall however it seems that Kant was still very orientated to the paradigm of the anthropocentric humanist view of XVII ${ }^{\text {th }}$ century of Pascal, Leibniz, Descartes, bacon and Newton, although an advocate of the criticism in the new Copernican revolution.

The XVII ${ }^{\text {th }}$ century saw countless developments along the path towards the future concept of computation, for instance Leibnizian binary code, machine enterprises such as Le Pascaline, the idea of modern age disembodiment of soul and body by Descartes, the scientific strongly inductive method of bacon under the auspice of commanding nature in action. Nevertheless, we conclude that the root of these developments was still predominantly anthropocentric and humanist, and this was the case until shortly post-industrialization.

This paper seeks to show that we ought to differentiate two traditions: the calculus and the computus. Turing's 1936 conception of computability unlocked the future basis for artificial intelligence. The idea of computare by means of artificial intelligence, on the lines of Turing's concept, has somehow a distinct imprint, effect and influence than that of calculus which was discovered simultaneously by Newton and Leibniz. I believe that this may partly have been due to the fact that Turing was an Englishmen, wandering in Sherbone and Cambridge in difficult times, and lived at the time the British empire, the biggest history has known, was crumbling and of the struggle for his nation's survival in war ii.

There is as much Leibniz in Gödel as there is of jacquard in 
Turing. Calculus is the representation of man alone just as computus is the exemplar of machinery. This seemingly innocent shift or relocation is sufficient, though, to alter consistently culture and civilization, or as Kant would have written, the metaphysical foundations of natural science. It is sufficient to alter, in other words, the notion of rule.

In order for functions to really function, the calculus paradigm had to be replaced by that of computus.

The progressive move from anthropocentric humanism, with so many narcissistic wounds inflicted to man since Galileo and the renaissance, to the loss of humanism in Turing's time is hard to follow, though we can say that technology is an over-riding concept that continues to become more and more important, matching the move from calculus to computus. Just as man alienated himself from thinking, in shifting from calculus to computus, some argue, so some argue that man was alienated from himself. With the passage of time one could say, on the contrary, that history reworks itself. One can cite the subsequent remark by Francis bacon, which emphasizes reasoning over over-confident and sometimes deceptive human memory, bearing in mind Turing's original idea of " $m$-configurations":

"(...) we shall analyze experience and take it to pieces (...) [3]."

\section{B. Turing's "Effective Calculable" Humanism}

Before closing this first section, we will embrace advances in relation to the notions we postulated at the start, i.e., function, rule, and algorithm. The intrinsic nature of such advances will be compared before we turn more exclusively to Turing in section two of this paper.

What the famous Babylonian clay tablet $Y B C 7289$ (Fig. 1) indicates to us is a square with diagonals drawn so that a correspondence of numbers from sides to diagonals could be devised, i.e., a coefficient of variables; in the same way, ancient Egyptians knew what we now know by $\pi$ as the relation of a circumference of a circle to its diameter by means of a ratio.

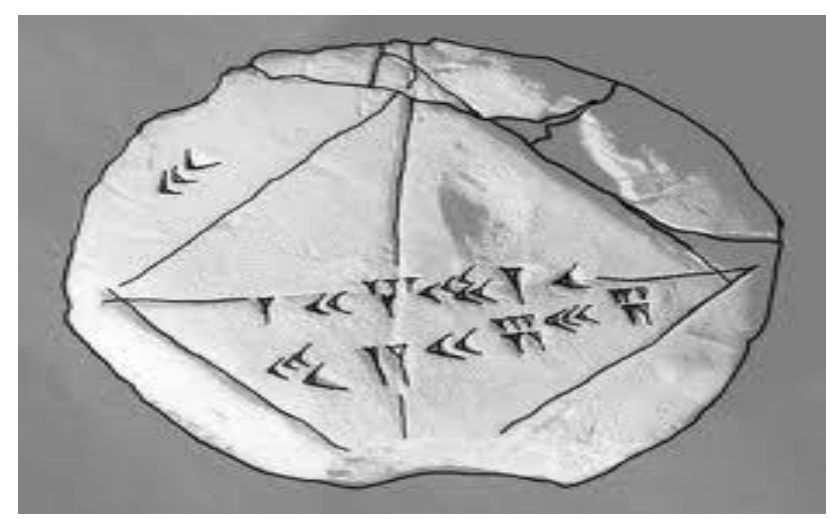

Fig. 1. The Babylonian clay tablet $Y B C 7289$.

The Pythagorean theorem was also of such nature that it served as a priori proposition that permitted one to see an established truth or rule independently, say, of the size or location of the triangles. Another example is the calculation that predicted eclipses of the sun.

There was a widely accepted notion of a curve along which different variables could relate to each other by means of an underlying rule. Arithmetic only developed into algebra at a later time, so the apparatus of formulae and strings of symbols had not yet encompassed the notion of a function. Even before Leibniz coined the term function in a rather adventurous prosaic epistolary style, of course there was a general perception of the bridge between one argument to one value considered to be a 'function'.

"In 1694 German mathematician Gottfried Wilhelm Leibniz, codiscoverer of calculus, coined the term function (Latin: Functio) to mean the slope of the curve, a definition that has very little in common with our current use of the word. The great Swiss mathematician Leonhard Euler (1707-83) recognized the need to make the notion of a relationship between quantities explicit, and he defined the term function to mean variable quantity that is dependent upon another quantity. Euler introduced the notation $f(x)$ for "a function of $x$," and promoted the idea of a function as a formula. He based all his work in calculus and analysis on this idea, which paved the way for mathematicians to view trigonometric quantities and logarithms as functions. This notion of function subsequently unified many branches of mathematics and physics. (...) advanced texts in mathematics today typically present all three definitions of a function — as a formula, as a set of ordered pairs, and as a mapping — and mathematicians will typically work with all three approaches [4]."

Unhesitatingly we also cite the large discoveries in realm of mathematics, such as that of Oresme, responsible for the first graph (or pictorial function, so to speak) or Napier and Briggs, who worked on tables of logarithms and machinery applications. It's really one all-embracing subject, but in my opinion, the first instances of this tendency go back to the renaissance cosmological vision as old as $\mathrm{XV}^{\text {th }}$ and $\mathrm{XVI}^{\text {th }}$ centuries, with Cusa and Bruno, and philosophies that were hospitable to the notion of Omnia Relata Est, without which the notion of function could have not given birth. And so, at this point, we are ready to settle our final conclusions of wide conceptual philosophical relatedness between function, rule and algorithm.

Having set out the concept of function, what it interesting is how the notion of algorithm as a list of procedures to approximate and resolve a function was crafted into computer science through the design of Turing machines and programming. In this sense, declaratively and procedurally, the function had to undergo the passage from a calculus paradigm to one of computus, so that a function could function.

But we shall not forget the slope of the curve referred to earlier in this paper. We come to a close by showing how human reason, on common logic grounds, complete to the systematic catalogue of operations it unfolds, by any other means except furnished by experience, is capable of demanding how far it can go, aiming at certitude and clearness, to the matter of critical enquiry of reason. It hesitates in a twofold relation of antinomies, just as similar of that exposed between theoretical and practical cognition. As said, it is a curve of a special tension, which we can use to correlate humanism with function.

This is essentially what permitted transcending perspectives about existing philosophical concepts and the bold and daring programme of $\mathrm{Ai}$ in computer science in the $\mathrm{XX}^{\text {th }}$ century. Human bio-machinery was also connected, 
through a kind of connectionism, with the concept of algorithm.

Just as critical reason was elaborated by Kant, we verify that Leibniz meant by function the slope of the curve and the limit, in Kantian, terms, therein constructed.

This was to produce a sort of natural deduction table from arithmetic to algebra, conformably proper to the schema of numbers to reality. This would be described in descending and ascending perception of quantities, with degrees of continuous generation. Indeed, it is of major importance how the term rule migrated to artificial intelligence and belongs nowadays to the jargon of programming languages.

We can now introduce the notion set out by Turing himself in his Princeton Ph.D. Thesis, that of functions being 'effectively calculable', as recalled by Andrew Hodges:

"A function is said to be 'effectively calculable' if its values can be found by some purely mechanical process. Although it is fairly easy to get an intuitive grasp of this idea, it is nevertheless desirable to have some more definite, mathematically expressible definition. Such a definition was first given by Gödel at Princeton in 1934... These functions were described as 'general recursive' by Gödel... Another definition of effective calculability has been given by church.. Who identifies it with lambda-definability. (...) We may take this statement literally, understanding by a purely mechanical process one which could be carried out by a machine..." [5]

By this mean Turing could through a function approach, fully and clearly articulate how effective calculability in the history of mathematics considered as one argument, could, by means of machinery and computation, reach different values, that is, a list of decimals, so that computation and calculability became as one. Turing fused, like jacquard's or Babbage's engines interweaved webs of textiles, in intellectual philosophical terms, the tradition of calculus with that of new emerging machinery powered by electricity most important above all, to that of computus.

Calculus and computus are, therefore, all explicitly joined, as are the concepts and dynamics of function, rule and algorithm, through Turing's all merit and extraordinarily genius.

No wonder too that this ended up as the information era, backed by the growing computation power of Moore's law, an exponential growth curve, passive of various anthropological interpretations.

\section{COMPUtus AbBreviated TABLE}

\section{A. Life in the Cell and in the Square}

We shall now concentrate on the paper on computable numbers with application to the Entscheidungs problem (1936) by Turing. Our discussion will, nevertheless, be linked to other insights. We cannot dismiss the fact that through the idea of the universal Turing machine doors were opened in all fields of knowledge, most notably, beyond technicalities in the core of computation, in philosophy of mind and affiliated fields. We have understood how, in the shift from calculus to Computus, the many-squares or multi-'dimensional' tables of calculus were reduced to a one-dimensional table where squares of symbols finitely run through.
This reduction was of a logarithm exponentiation kind. The insight about the universal Turing machine is precisely this intangible asset. So too in law and moral philosophy the extraordinary role of constitutions is one balance between reduction in things and exponentiation of ideas and liberties.

Just as "pairs" were to be one possibility of devising Functions, as seen above, so we find in Turing's paper:

"The possible behaviour of the machine at any moment is determined by the $m$-configuration $\mathrm{q}$ and the scanned symbol $s(r)$. This pair $q, s(r)$ will be called the 'configuration': thus the configuration determines the possible behaviour of the machine." [6]

The so called universal Turing machine is only depicted in section 6 of the paper, when Turing aims only to describe computing machines. Turing has an interesting view about number theory, as he considers expressivity instead of classes or sets. It is the expression of variables in terms of computer numbers that he targets, but this does not mean that they are not concrete functions or real by principle. This topic is not to be underestimated, as since antiquity we have called naturals the class of numbers which are most easily intuited in empirical terms, and now Turing followed a path that is more Expressivist than essentialist or platonic. His approach provided insightful technical details, of both theoretical and machinery solutions, which bonded calculus to decimals, decimals to computus, and, thus, in perspective, calculus to computus.

Decimals are just one way of expressing numbers, a least expensive currency of numbers, so to speak. Turing also discusses the approach of 'predicates', which can be extended outside the realms of traditional number theory, by resorting to understanding exactly how expressivity of numbers is just about predication inside number theory.

Turing would have agreed with the proposition that numbers are just one limited example of mathematical predicates (they do not directly entail conceptual framework, such as "divisibility", "primality", “ideals", "greatest common Divi- sors" or "unique factorization") but in such neutral limitation, of akin kind of human's memory limitation, they have the power to resort functions so complex that they surpass its natural limits, equally as man was, in spite of its 'short memory', capable of industrializing primes in tables and, ultimately, capable of resorting different theoretical and practical choices in the very difficult even slope of antinomies. That's why Turing, rather provocatively uses the adverb "naturally" in the expression:

"(...) all numbers which could naturally be regarded as computable [6]."

We must understand that the class of computable numbers is enumerable, but it does not include all definable numbers. Philosophically, that the class of definable numbers is non-congruent (in gauss' terms) with enumerable numbers is very interesting and close to Gödel's legacy. Analyzing computation principles, the reader is confronted with the paradox of infinity. Where can we find non-definable numbers if "number" is basically a pointwise definition, basically restricting a quantity to some unity, at least according to Newton's classical "definition"? This is similar to berry's paradox: "the smallest possible integer not definable by a given number of words". 
In Gödel's work, infinity is a thing to marvel at, with so much ineffable relations. Turing is very aware of this, and so he say his results approach Gödel's. Turing says too that the Hilbertian Entscheidungs Problem cannot have a solution. Nevertheless, as this is one important element to follow, we call attention to the comparison: just as one function draws a graph or curve and designs a top and bottom field of values, originally itself the meaning of function - the subfield below the curve to be more precise - so too with computation we have outlined above, in Gödel's fashion, the unsolvable Hilbert problem, and below Alonzo's "effective calculability" equivalent to Turing's "computability".

The genius of Turing was to envisage the empowerment out of the vanishing belief in mathematics after the incompleteness theorem let out by Gödel. Turing was to discover the sound basis for incompleteness in the almost complete set of computable numbers, and this reverse approach to the Hilbertian problem is, in fact, one excel demonstration of the creative power of imagination. This new engineering shift and vision led to many problem solving techniques, instead of halting stifling investigation of the Entscheidungs Problem.

In Section I. Named "computing machines" Turing without any trace of shame, and at a time in history when many were reluctant to do so because humanism was under attack by Faustian belief in technology, adhered to the view that impudently attacked the humanist belief most profound out of all, which is anthropocentrism. He inflicted another narcissistic wound on man, by affirming the comparison between the human brain and computing machines, even though under the acceptance of computation as one extension of humankind.

There is, inarguably, a very strong congruence effect from bios to computus, dragging the same colonizing effect of life in the cell and in the square. This corresponds to an $\mathrm{Ai}$ argument thoroughly explored by Dennett, for instance, when reasoning about Darwin's legacy and contemporary philosophy, even though in Turing's case his mor- phogenesis books are often in the shadows. This is why maybe Turing is thinking about humans too, and not only human intervention as constituent of one external factor in choice-machines ( $c$-machines) to the goal of insinuating that the human species is, precisely, a $c$-machine.

Having in mind to make things from what is commonest in nature and with the least waste of energy, Turing reached a path designed desirably so much for bios as for computus.

"A sequence is said to be computable if it can be computed by a circle-free machine. A number is computable if it differs by an integer from the number computed by a circle- free machine [6]."

By developing a model in this way, both the human mind and machinery, what Turing is saying is that nature, as a field of calculus (in a restricted sense) encounters computus, so that nature itself can be interpreted as truth tables, better said Turing machines, being the infinite tape time, and nature (in the restricted sense of consciousness, and the synthesis of imagination) being the probabilistic multi-dimension squares.

There is also a powerful philosophical move here towards symbolism, as many symbols describe many discrete configuration states, which obscures the break from continuous calculus to integers and decimals. In fact, Turing exposes a major defect of the human mind, namely its lack of memory. He both enlarges and restricts in scope both artefacts: human mind and machinery.

We are told in many encyclopedia articles, for example, how Turing machines are in many ways more powerful than state of the art computers, since they are not restricted by any memory storage limitations. There are some variations in the model of a Turing machine, for example where one slides only to the right or ones possessing 5-tuple transitions basic states, instead of the classical 4-tuple transitions: (state, character $) \rightarrow$ (new state, new character $\bigvee$ direction), meaning that it is impossible to see what we find in so many printing robots, which print and move all in one move.

There is also a similarity of consequences comparing the inescapably insufficient computability and the self-inspection method of paradoxes when transposed to the problem of a universal Turing machine, namely with the so called "halting problem": one circle-free Turing machine, if analyzed by any other is prone to as much circularity as the continuum problem.

The "halting problem" is itself a paradox, as it happens by default in any circle-free Turing machine, and there is even a certain measure of choice for the machine to make when running, for example, when blank fields are encountered. As Turing stipulated in the paper, this behavior applies to operations on any symbol and also on no symbol. Turing used the example of the sort of palindrome " 010101 " to 4 " $m$-configurations" under alphabetical notation, in a manner that out of the four operations only two can print out one " 0 " and one " 1 " for each complete cycle and repeatedly, allowing only one "right" direction.

The philosophy of language has developed significantly due to reasoning through the work of Turing, independently of paradoxes. Language by its very nature can shelter computational m-configurations and intuitionist views. Poetry, for example, has attracted senior Germanophile philosophizing, like Goethe, Wittgenstein and Heidegger, and we cannot say that Gödel is not entitled to be out of this group.

Andrew Hodges gives details about how Turing changed his views on Abductionist and intuitionist views over time, now with the exception of being reversed. At the start he shared a similar to Gödel's and post's view when he wrote systems of logic based on ordinals (1939, i.e. The pre-war period), but in the later phase he became pro-engineering and computing-aware, and came up with new insights about definability and uncomputable queries (i.e. The war period). This is illustrated clearly in this passage by Hodge:

"Instead, he decided, the scope of the computable encompassed far more than could be captured by explicit instruction notes, and quite enough to include all that human brains did, however creative or original. Certainly, by the end of the war, he was captivated by the prospect of exploring the scope of the computable on a universal Turing machine; and indeed he called it 'building a brain' when talking of his plans to his electronic engineer assistant. For 1) it was conceived from the outset as a universal machine for which arithmetic would be just one application, and 2) Turing sketched a theory of programming, in which instructions could be manipulated as well as data [7]." 


\section{B. Nature as "abbreviated table"}

Let us recall that in our intellectual venture, we have sought to analyze, in the shift from the tradition of calculus to computus, precisely one conceptual reality that could have asked borrowed the term "abbreviated table". This interpretation contains also the seeds to his later conceptions in the paper the chemical basis of morphogenesis (1952), which Harbours a strong connectionist perspective, something that was indicted by contemporaneous philosopher of mathematics and $\mathrm{Ai}$, roger Penrose, as expounded by Hodges:

"The argument from continuity in the nervous system: the nervous system is certainly not a discrete-state machine. A small error in the information about the size of a nervous impulse impinging on a neuron, may make a large difference to the size of the outgoing impulse. It may be argued that, this being so, one cannot expect to be able to mimic the behaviour of the nervous system with a discrete-state system. (...)

But this brings us to Penrose's central objection, which is not to the discreteness of Turing's machine model of the brain, but to its computability. Penrose holds that the function of the brain must have evolved by purely physical processes, but that its behaviour is - in fact must be - uncomputable [8]."

The abbreviated "skeleton tables" as Turing calls them, even though they are not central to his argument, are nevertheless fundamental in the way they introduce firstly the expression " $m$-configuration function" or " $m$-function". This happens so precisely due to the "abbreviated factor". Symbols of the machine and $m$-configurations, being the only admissible expressions to be contained therein, are thus exposed so to virtually enable copying, comparing and sequencing symbols of any given form.

We say this so as not to close our investigation without demonstrating the role of functions as rules in the core of computus, demonstrating too that the curve of functions is a sort of infinite parallel between all domains, so to have made possible the bridge from calculus to computus. We can see this amongst its uncountable extensions, moreover expound to sound basis, of theoretical worlds so set apart as the continuum problem and the nervous system continuity, similar in all ways to the other pairs referred already, most notably other than Calculus to Computus, the passage from incompleteness to effective calculability. We can comprehend that there is some hidden meaning in the expression skeleton tables in relation with $m$-functions so to produce at the end the complete tables for the $m$ configurations, bearing in mind Turing's intellectual biography. "skeleton" is not just meant to signify a raw and incomplete form or declination table to the aim of producing $m$ configurations.

It conveys the idea of computing as a skeleton table for the bios, inasmuch as a skeleton holds a body and is emergently the in reductio most raw form of the astonishingly rich surrounding interface of the human body, the unique example amongst all vertebrates, namely having genius.

This is the point where implant technology is supposed to start, to demonstrate computing as it were as a prosthetics of bios and man, in Turing's words, "naturally", as if it was being said time prosthetic of space. Antinomies, first-order logic recursively axiomatic incompleteness and the continuum problem are just about the high-level problems to which correspond some low-level efficiency, as with effective calculability (the Turing machine idea behind computation) and now, in more utopian and prospective style by the argument from continuity in the nervous system, one idea that shaped greatly his later works and $\mathrm{Ai}$ as an application field of computer science.

In fact, Turing's approach enhances a very specific convergence, when the machine finds the symbol from the $m$ configuration farthest to one side, becoming any altered state depending on the finding of the symbol, as if it would represent, in theory, animats - animals and materials - or hybrots - hybrids and robots - which are circle-free approximations to both bios and computing. Curiously, the farthest convergence of cosmos with computing was not, to my knowledge, one debatable issue in Turing's mind, at least to have forced him to write about it consistently.

It seems that the curve of this problem, equivalent to the time of history, and equivalent to the discovery of functional analysis throughout history, apart from being intrinsic with human's perception of all things relatedness, is essentially related to the close gaping of circle-free Turing machines results. Here we have one paradox, thus: Turing's machine (in the limit Turing's postulated universal Turing machine), supposedly a circle-free machine, is, conversely, one halting problem in prospect, for the single reason that the circle between computation and cosmos is lessening more and more, and so is, to our era and following, the circle between computation and bios, at least accordingly to Turing's Ai disciples.

The teleological capacity of man is, thus, related to, in like manner, with antinomies, the continuum problem, first-order logic recursively axiomatic incompleteness, as with the halting problem of the universal Turing machine. Turing holds, without a doubt, the cosmos computing vision, not just a mere bios computing vision. One simple bioscomputing vision was supplemented, for instance, by some Enactivist accounts, besides simple connectionism, some sensorimotor theories of perception, or bios and cosmos semiotics impressions.

Turing transplanted one to the other representational views, action to cognition and environmental recognition views, just as the slope depicted under the idea of function aggregated different values through a rule.

We have speculated now of how much and to which extent morphogenesis could bias to cosmogenesis and in accordance to Turing's insights, but this is not the chief goal for this paper. Finding the concept of function inside computus with Turing, bringing the computing classical model to encounter its foundations in calculus, is one ascending historical curve too, and was this article's main goal, so to ascertain the faculty of imagining the synthesis behind. The aim was also to make an embryonic interpretation of abbreviated tables.

\section{CONCLUSION}

In conclusion, I hope to have explained the place of Turing machines in the history of ideas, exquisitely crafted so as to characterize, in Kantian terms, one image and object capable 
of, not only by analogy, unifying acts of recognition in discrete particulars, but, more extensively, to overpass the idea of one receptacle for foreign representations, and in continuum reproduce, as consciousness presented to oneself, by the gift of the faculty of imagining, one thoroughgoing synthetic unity, working for the whole of knowledge. Turing, to whom with so much pleasure we have just celebrated a centenary of life and work, has inspired man to all its imaginable and unimaginable heights.

\section{ACKNOWLEDGMENT}

My personal thanks go to the generosity of Mr. Ian Thomson.

\section{REFERENCES}

[1] I. Kant, The Critique of Pure Reason, Transcendental Doctrine of Elements, Section I, of Space, Conclusions from the Foregoing Conceptions Kant's Critiques, $1^{\text {st }}$ ed., Wilder Publications, 2008, pp. 40-41.

[2] I. Kant, "The critique of pure reason," in (A 159/B198)," in The Cambridge Companion to Kant and Modern Philosophy, Paul Guyer, Ed. Unversity of Pennsylvania, Cambridge University Press, 2006, pp. 47.

[3] F. Bacon, "The plan of Instauratio Magna," The Arguments of Several Parts, 11 in Prefaces and Prologues, vol. 39, pp. 1909-1914, 2001.

[4] J. Tanton, Encyclopedia of Mathematics, History of Functions, New York: Facts on File, pp. 208

[5] A. Turing, "Systems of logic based on ordinals," in Proc. Lond. Math. Soc., vol. 45, no. 2, 1939, pp. 161-228.
[6] A. M. Turing, "On computable numbers, with an application to the Entscheidungs problem" Proc. London Math. Soc., 1936, vol. 2, no. 42, pp. $230-265$

[7] A. Hodges. (1997). "The impact of wartime work on Turing's philosophy." Alan Turing, One of the Great Philosophers. [Online] Available: http://www.turing.org.uk/publications/ex6.html

[8] A. Hodges. (1997). "The uncomputable revisited." Alan Turing, One of the Great Philosophers. [Online]. Available: http://www.turing.org.uk/publications/ex11.html

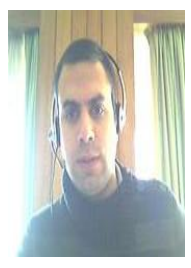

Luís Homem was born in Lisbon on December 21, 1978. He graduated in philosophy in the Faculty of Letters of the University of Lisbon in 2005. He concluded a master in Natural and Environmental philosophy in the same Faculty with the Thesis "The New Plasticity on Time and Analytics in Darwin" in 2009. He also completed a master in Logic and philosophy of Science in the University of Salamanca in 2012 with the Thesis "Topics in Programming Languages, a Philosophical Analysis through the case of Prolog." He is currently completing his doctoral thesis in Salamanca.

Luís Homem is an integrated member of the Centre for Philosophy of Science of the University of Lisbon since the summer of 2011 and has in the past years dedicated great attention to Artificial Intelligence domains, varying its study from Natural philosophy topics to Programming Languages and Modelling. He devotes still great deal of his time to Classical and Contemporaneous philosophy and strives to produce investigation in those areas as well. Some of his key publications are Topics in Programming Languages (Oxford: Chartridge Books Oxford, 2013) and "Naturalizing Prolog" in Ensayos sobre Lógica, Lenguaje, Mente y Ciencia, VI Jornadas Ibéricas, Alfar Sevilla, 2012, pp. 31-47. He has one son, named Afonso, who is the joy of his life, through whom blessing flows and Past and Future are seen. 